MAT

$1.994 / 1087 \mathrm{een} \cdots$

$1993 / 05 / 11$

\section{Weight-balanced measures and free energy for one-dimensional dynamics}

Artur O. Lopes and Wm. Douglas Withers*

(Communicated by Giovanni Gallavotti)

Abstract. In this paper we consider Thermodynamic Formalism properties of one dimensional maps. We consider the existence of weight-balanced measures and large deviation properties of the Free-Energy of the Jacobian of measures. We show that a weight-balanced measure exists under the hypotheses that the map is piecewise-homeomorphic and the weights piecewise constant.

We consider also a certain class of measures with the property that the Free-Energy of the Jacobian is differentiable by parts. For measures in this class we show that a certain measure is the maximal entropy measure if and only if the Free-Energy of the Jacobian is linear. The result follows from general properties of Large-Deviation Theory and does not use the more classical approach of Thermodynamic Formalism.

1991 Mathematics Subject Classification: 58F11.

\section{Introduction}

In this article we consider a dynamical system consisting of a continuous function $f$ on an interval $[a, b]$ with the following properties:

(i) $f([a, b])=[a, b]$.

(ii) There exist points $a=c_{0}<c_{1}<\ldots<c_{d}=b, d \in \mathbb{N}$, such that $f$ is homeomorphic on $\left[c_{i}, c_{i+1}\right], i \in \sigma=\{0,1, \ldots, d-1\}$, but $f$ is not one-to-one in any neighborhood of $c_{i}, i \in \sigma-\{0\}$. For example, if $f(x)=\lambda x(1-x), \sqrt{5}+1<\lambda \leq 4$. $f$ satisfies these hypotheses if we take $d=2, c_{1}=1 / 2, b=c_{2}=f\left(c_{1}\right)$. and $a=c_{0}=f\left(c_{2}\right)$.

*Partially supported by the Office of Naval Research 
We say that a map $f$ is expanding if $f$ is continuously differentiable and there exist $\alpha>0, \lambda>1$ such that $\left.\left|\left(f^{n}\right)^{\prime}(x)\right|>\alpha\right\rangle^{n}$ for all $n \in \mathbb{N}$ and all $x$ in the nonwandering set (see Mañe [15] for definitions). The nonwandering set is a Cantor set in this case.

In some aspects, a real quadratic map has more obscure dynamics than a complex quadratic map. A major difficulty is that not all points have the same number of preimages under a real quadratic map. While the entropy of a real quadratic map is given by the exponential rate of growth of the number of preimages of the critical point (see Misiurewics [16] and Misiurewics and Slenk [17]), finding the measure of maximal entropy is more difficult in the real case. Moreover, Hofbauer [8] showe that while a real quadratic map has a unique measure of maximal entropy, a real cubic map can have more than one measure of maximal entropy.

This contrasts with the case of a complex rational map $g$ of degree $d>1$, for which the entropy is always given by $\log d$ and the measure of maximal entropy always exists uniquely.

The maximal entropy measure is the weak limit as $n$ goes to infinity of the measure giving mass $d^{-n}$ to each of the $d^{n}$ points $g^{-n}\left(z_{0}\right)$. Here $z_{0}$ can be any fixed point of $\mathbb{C}$ with at most two exceptions. The Jacobian of this measure is simply $1 / d$. Finding the Jacobian of a maximal entropy measure for a real polynomial map is much more difficult.

If we try to proceed in an analogous way for a real quadratic polynomial, assigning equal masses to the preimages of the critical point, we do not cven obtain an invarian measure in the limit. Again, this is because various points may have one, two, or no preimages under $f$. This article was partly motivated by the problem of suitably assigning masses to the preimages of a point in order to obtain a maximal-entropy measure in the limit. The procedure defining a measure by assigning masses to preimages of a point is useful in determining a functional equation satisfied by the moment-generating function of the measure. The solution to this problem is still unknown, even in the case where $f$ is expanding.

An invariant measure created by assigning different masses to the preimages of a given point is essentially a weight-balanced measure. Barnsley and several other authors [2], [5], [6]. [12] consider weight-balanced measures that are essentially measures obtained from balanced probabilities (see [5] for references). These balanced probabilities are related to the Jacobian of such measures ([12]). The definition of the Jacobian of a measure is also given in Section 2.

An important problem in the theory of weight-balanced measures is that of finding a measure with a predefined Jacobian. This type of measure is also sometimes called a $g$-measure. Several interesting results in this direction have been obtained [2]. [5] [6]. Barnsley, Elton. Demko, and Geronimo [2] is a general reference about the subject. Most of these results assume the map has a fixed number of preimages. In this article we study properties of weight-balanced measures for (not necessarily polynomial) maps satisfying hypotheses (i) and (ii) given at the beginning of this section. This study differs from previous work on weight-balanced measures in allowing the number of preimages to vary from point to point.

In Section 1 we consider the existence of weight-balanced measures for such maps. not necessarily expanding or even differentiable, with associated piecewise-constant probabilities (Theorem 1.7). In Section 2 we study properties of the free energy of the Jacobian of an invariant measure under the hypotheses that the measure has Jacobian with Free-Energy differentiable by parts.

One of the results presented in the present paper is that a measure is the maximal-entropy measure if and only if the free energy is linear (Theorem 2.2).

Using this result in the case where $f$ is expanding we characterize maximal-entropy measures as those whose Jacobians have zero asymptotic variance. These results are related to some previous ones of Zieman [28. Theorem 3].

We hope some of these techniques can be applied to obtain existence of measures with densities under summability conditions as in [19].

A large part of the results in Section 1 were already known ([1]. [9]. [23]). We learned this fact after our paper was written. The proof presented here is new and uses different ideas that we believe can be applied to more general situations.

Our main result in Section 2 is Theorem 2.2 where we do not assume expansiveness. The proof does not use the Perturbation Theory of Linear Operators ([1]. [9]. [23]) but general properties of Large-Deviation Theory. We believe these techniques can be applied in very general situations to give a general characterization of the maxima entropy measure.

Note that the Ruelle-Perron-Frobenius operator can be applied in our situation (with varying numbers of preimages) in the expanding case. The Ruelle-PerronFrobenius operator acts on Hölder-continuous functions $\phi$ defined on the nonwandering set and is given by

$$
L_{\psi}(\phi)(x)=\sum_{f(y)=x} \phi(y) e^{\Psi_{(y)}} .
$$

$\psi$ being some Hölder-continuous real-valued function defined on the nonwanderin set. Using the same reasoning as when the number of preimages is fixed. one can show there exists a Hölder-continuous eigenfunction $\phi^{*}$ such that $L\left(\phi^{*}\right)=e^{h+1} \phi^{*}$. where $h(f)$ is the entropy of $f$ (see Ruelle [22]). The problem is that it is not easy to solve this functional equation. Knowing $\phi^{*}$, we could find the Jacobian of the maximalentropy measure $v$ as

$$
J_{v}(x)=\frac{\phi^{*}(x)}{e^{h(J)} \phi^{*}(f(x))} ;
$$

and this shows that $\log J_{v}(x)$ is cohomologous to $h(f)$ and hence has zero asymptotic variance. We show in this article that $\log J_{v}(x)$ has zero asymptotic variance using other methods (Theorem 2.6). This also shows that $J_{v}$ is Hölder-continuous.

Note also that the free energy can be considered as the definition of the generalized entropy, as is done in Lopes [11]. This concept was introduced and discussed in several physics papers, but for rigor we prefer an approach borrowed from Large-Deviation Theory.

We would like to thank K. Zieman and M. Viana for some helpful conversations about one-dimensional dynamics. 


\section{The existence of a weight-balanced measure}

We consider continuous functions $f$ on an interval $[a, b]$ with the following properties:

(i) $f([a, b])=[a, b]$.

(ii) There exist points $a=c_{0}<c_{1}<\ldots<c_{d-1}<c_{d}=b$ such that $f$ is homeomorphic on $\left[c_{i}, c_{i+1}\right], i \in \sigma=\{0, \ldots, d-1\}$, but $f$ is not homeomorphic on any neighborhood of $c_{i}, i \in \sigma-\{0\}$.

We define $C$ to be the set $\left\{c_{1}, \ldots, c_{d-1}\right\}$. We set

$$
\begin{aligned}
& E=f(C)-\{a, b\}, \\
& F=\bigcup_{n=0}^{\infty} f^{n}(E) .
\end{aligned}
$$

The set $E$ has no more than $d-1$ elements.

For each $i \in \sigma, f$ is a homeomorphism when restricted to $I_{i}=\left[c_{i}, c_{i+1}\right]$; we denote the inverse of the restriction of $f$ to $I_{i}$ by $\beta_{i}: f\left(I_{i}\right) \rightarrow I_{i}$.

(iii) We also consider functions $p_{i}:[a, b] \rightarrow[0,1], i \in \sigma$ such that $p_{i}(x)>0$ if and only if $x \in f\left(I_{i}\right), p_{i}$ is piecewise constant with discontinuities only at points of $E$, and

$$
\sum_{i \in a} p_{i}(x) \equiv 1 .
$$

We set $p=\left(p_{0}, \ldots, p_{d-1}\right)$

We define a Borel measure $\mu$ on $[a, b]$ to be $p$-balanced if

$$
\mu \beta_{i}(X)=\int_{X} p_{i}(x) d \mu(x)
$$

for all $i \in \sigma$ and Borel subsets $X$ of $[a, b]$. Equivalently, $\mu$ is $p$-balanced if

$$
\int \phi d \mu=\int T \phi d \mu
$$

for all continuous $\phi:[a, b] \rightarrow \mathbb{R}$, where we define the operator $T$ by

(1.A) $T \phi(x)=\sum_{i \in \sigma} p_{i}(x) \phi\left(\beta_{i}(x)\right)$.

Since $p_{i}(x)=0$ unless $x$ is in the domain of $\beta_{i}, T$ is well defined.

We say $\mu$ is invariant under $f$ if $\mu f^{-1}(A)=\mu A$ for any Borel subset $A$ of $[a, b]$. Note that weight-balanced measures are always invariant. It is also true that

$$
\int \phi \circ f(x) d \mu(x)=\int \phi(x) d \mu(x)
$$

for any integrable function $\phi$ and any invariant measure $\mu$.

We denote by $\%$ the operation of concatenation from $\sigma^{k} \times \sigma^{n}$ to $\sigma^{k+n}$.

$$
\left(i_{1}, \ldots, i_{k}\right) \mid\left(i_{k+1}, \ldots, i_{k+n}\right)=\left(i_{1}, \ldots, i_{k+n}\right) .
$$

We can then write

$$
T^{k} \phi(x)=\sum_{i \in \pi^{k}} p_{i}(x) \phi\left(\beta_{i}(x)\right) .
$$

where $p_{i}$ and $\beta_{i}$ are defined recursively by $\beta_{i \mid j}(x)=\beta_{i}\left(\beta_{j}(x)\right)$, and

(1.B) $\quad p_{i \mid j}(x)=p_{j}(x) p_{i}\left(\beta_{j}(x)\right)$.

Note that $p_{i}(x)>0$ if and only if $x$ is in the domain of $\beta_{i}$.

We assume one additional hypothesis:

(iv) There exists $K$ such that

$$
P=\sup _{x \in[0, b)} \sup _{i \in \sigma^{\kappa}} p_{i}(x)<1 \text {. }
$$

This is equivalent to saying that each $x \in[a, b]$ has at least two preimages under $f^{\boldsymbol{k}}$.

Our goal in this section is to prove the existence and uniqueness of a $p$-balanced probability measure for a map $f$ and probabilities $p_{i}$ satisfying hypotheses (i)-(iv).

\subsection{Lemma. Let}

$$
\operatorname{disc} p_{i}(x)=\left|p_{i}(x)-\lim _{y \rightarrow x^{-}} p_{i}(y)\right|+\left|p_{i}(x)-\lim _{y \rightarrow x^{+}} p_{i}(y)\right|
$$

be the discontimuity of $p_{i}$ at $x$. Then

$$
\sum_{i \in \sigma^{k}} \operatorname{disc} p_{i}(x)=0
$$

unless $x=f^{n}(e)$ for some $e \in E$ and $0 \leq n<k$. Moreover. there exists a constant $M$ independent of $k$ such that if $x=f^{n}(e)$ for some $e \in E$ and $0 \leq n<k$.

$$
\sum_{i \in \sigma^{k}} \operatorname{disc} p_{i}(x) \leq M \prod_{j=1}^{n} p_{m j-1}\left(f^{j}(e)\right)
$$

where we choose $m_{j} \in \sigma$ so that $f^{j-1}(e)=\beta_{m_{j-1}}\left(f^{j}(e)\right)$

Proof. To prove the first part of the lemma, we use induction on $k$. The case $k=1$ is mmediately obting lemma holds up to $k-1$ For $i \in \sigma^{k-1}$, we have $p_{i i_{k}}(x)=p_{i_{k}}(x) p_{1}\left(\beta_{i_{k}}(x)\right)$. If $\operatorname{disc} p_{i \mid i_{k}}(x)>0$ then either $\operatorname{disc} p_{1}\left(\beta_{i}(x)\right)>0$ or $\operatorname{disc} p_{i_{x}}(x)>0$. If $\operatorname{disc} p_{i}\left(\beta_{i_{k}}(x)\right)>0$, then by assumption we disc $\rho_{i}\left(\beta_{i k}(x)\right)>0$ or $f^{n}$, fisere $x=f^{n+1}(e)$. If have $\beta_{i_{k}}(x)=f^{n}(e)$ for some $e \in E$ and $0 \leq n<k-1$, therefore $x \in E$.

disc $p_{i_{k}}(x)>0$ then the first part of the lemma holds because $x \in E$.

To prove the second part of the lemma, we let $F_{1}$ be the set of all points $x$ of $F$ such that $x \in E$ or the set $f^{-1}(x) \cap F$ has more than one point. Note that, while $F$ may be 
infinite, $F_{1}$ must be finite. Let $k_{1}$ be large enough that, for any $x \in F_{1}$, there exists $e \in E$ and $n<k_{1}$ such that $x=f^{n}(e)$. Set

$$
M=\sup _{e \in E} \sup _{k \leq k_{1}, 0 \leq n<k} \sup _{0 \in n^{*}} \sum_{i \operatorname{sisc}} p_{i}\left(f^{n}(e)\right) \text {. }
$$

This ensures that the second part of the lemma holds for all $k \leq k_{1}$ and in particular holds for all cases where $x \in E$ or $f^{-1}(x) \cap F$ contains more than one point. We now work again by induction. Suppose the second part of the lemma holds up through $k-1 \geq k_{1}$. For $i \in \sigma^{k-1}$, we have $p_{i \mid i_{k}}(x)=p_{i_{k}}(x) p_{i}\left(\beta_{i_{k}}(x)\right)$. Thus

$$
\operatorname{disc} p_{i \mid i_{k}}(x) \leq p_{i_{k}}(x) \operatorname{disc} p_{i}\left(\beta_{i_{k}}(x)\right)+p_{i}\left(\beta_{i_{k}}(x)\right) \operatorname{disc} p_{i_{k}}(x)
$$

The second part of the lemma follows immediately from the first unless $x=f^{n}(e)$ for some $c \in E$ and $0 \leq n<k$; let us therefore assume that this is so. Moreover, our choice of $M$ yields the desired conclusion if $x \in E$ or $f^{-1}(x) \cap F$ has more than one point; let us therefore assume that $x \notin E$, and that $f^{-1}(x) \cap F$ has only one element. Then $\operatorname{disc} p_{i_{\mathrm{k}}}(x)=0$, and

$$
\operatorname{disc} p_{i \mid i_{k}}(x) \leq p_{i_{k}}(x) \operatorname{disc} p_{i}\left(\beta_{i_{k}}(x)\right) .
$$

Thus

$$
\begin{aligned}
\sum_{i \mid i_{k} \in \sigma^{k}} \operatorname{disc} p_{i \mid i_{k}}(x) & =\sum_{i_{k} \in \sigma \in \sigma} \sum_{i \in \sigma^{k-1}} \operatorname{disc} p_{i \mid i_{k}}(x) \\
& =\sum_{i_{k} \in \sigma}\left(p_{i_{k}}(x) \sum_{i \in \sigma^{k-1}} \operatorname{disc} p_{i}\left(\beta_{i_{k}}(x)\right)\right) .
\end{aligned}
$$

Let $m_{n-1} \in \sigma$ be chosen so that $f^{n-1}(e)=\beta_{m_{n-1}}\left(f^{n}(e)\right)$. Since we are assuming that $f^{-1}(x) \cap F$ has only one element, and the first part of the lemma ensures that all the discontinuities of $p_{i}$ lie in $F$, we can have disc $p_{i}\left(\beta_{i_{k}}(x)\right)>0$ only if $i_{k}=m_{n-1}$. Thus

$$
\sum_{i \mid i_{k} \in \sigma^{k}} \operatorname{disc} p_{i \mid i_{k}}(x)=p_{m_{n-1}}(x) \sum_{i \in \sigma^{k-1}} \operatorname{disc} p_{i}\left(\beta_{m_{n-1}}(x)\right) .
$$

By assumption,

$$
\sum_{i \in \sigma^{k-1}} \operatorname{disc} p_{i}\left(\beta_{m_{n-1}}(x)\right)=\sum_{i \in \sigma^{k-1}} \operatorname{disc} p_{i}\left(f^{n-1}(e)\right) \leq M \prod_{j=1}^{n-1} p_{m_{j-1}}\left(f^{j}(e)\right) .
$$

Thus

$$
\sum_{i \mid i_{k} \in \sigma^{k}} \operatorname{disc} p_{i i_{i}}(x) \leq M \prod_{j=1}^{n} p_{m_{j-1}}\left(f^{j}(e)\right)
$$

1.2 Lemma. If

$$
D=\bigcup_{n=0}^{\infty} f^{-n}(C)
$$

is dense in $[a, b]$, then the family

$$
\left\{\phi, \beta_{i}: i \in \sigma^{k}, k=1,2, \ldots\right\}
$$

is uniformly equicontimuous for any contimous $\phi$ on $[a, h]$

Proof. Let $\varepsilon>0$ be given. We must find $\delta>0$ such that

(1.C) $\left|\phi\left(\beta_{i}(x)\right)-\phi\left(\beta_{i}(y)\right)\right|<\varepsilon$

for all $|x-y|<\delta$ and all $k$ and $i \in \sigma^{k}$. Choose $\theta>0$ so that $|\phi(x)-\phi(y)|<\varepsilon$ for $|x-y| \leq \theta$. Choose $m$ sufficiently large that any point in $[a, b]$ is within $\theta / 2$ of $\beta_{i}(c)$ for some $c \in C, k \leq m, i \in \sigma^{k}$. Then (1.C) holds for any $k>m$. any $i \in \sigma^{k}$. and any $x, y \in[a, b]$ for the following reason. If $k>m, i \in \sigma^{k}$, then the range of $\beta_{i}$ can contain no point $\beta_{j}(c)$ for $c \in C, n \leq m, j \in \sigma^{n}$. Thus $\operatorname{diam} \beta_{i}([a . h]) \leq \theta$.

The finite family

$$
\left\{\phi \circ \beta_{i}: k=1, \ldots, m, i \in \sigma^{k}\right\}
$$

is uniformly equicontinuous; we can thus choose $\delta$ so that (1.C) holds for all members of this family. $\square$

1.3 Theorem. If $D$ as defined in Lemma 1.2 is dense in $[a, b]$, then there exists a p-balanced probability measure $\mu$ for $f$.

Proof. We show that $T^{k} \phi$ converges uniformly for any continuous real-valued $\phi$ on $[a, b]$. The $p$-balanced measure $\mu$ can then be defined by

$$
\int \phi(x) d \mu(x)=\lim _{x \rightarrow \infty} T^{k} \phi(y)
$$

for some fixed $y \in[a, b]$.

The first step is to prove that

(1.D) $\sum_{n=1}^{\infty} \prod_{j=1}^{n} p_{m_{j-1}}\left(f^{j}(e)\right)<\infty$

for all $e \in E$, where $m_{j-1}$ is chosen so that $f^{j-1}(e)=\beta_{m_{j-1}}\left(f^{j}(e)\right)$. Note that

$$
\prod_{j=1}^{n} p_{m_{j}}\left(f^{j}(e)\right)=p_{\left(m_{0} \ldots \ldots m_{n-1}\right)}\left(f^{n}(e)\right)
$$

From hypothesis (iv), we have $p_{i}(x) \leq P^{n}$ for $i \in \sigma^{n k}$. Thus the sum in (1.D) is dominated by a geometric series and hence converges.

We would like to point out that the above theorem can be stated in a more general from with the only assumption that (1.D) is true. 
For $x \in F$, set

$$
q(x)=\inf _{e, n} \prod_{j=1}^{n} p_{m_{j-1}}\left(f^{j}(e)\right),
$$

where $e \in E$ and $n \geq 0$ such that $x=f^{n}(e)$. We define mappings $\pi_{-1}, \pi_{0}$, and $\pi_{1}$ on $[a, b]$ by

$$
\begin{aligned}
& \pi_{-1}(x)=x+\sum_{y \in[a, x) \cap F} 2 q(y), \\
& \pi_{1}(x)=x+\sum_{y \in[a, x] \cap F} 2 q(y),
\end{aligned}
$$

and

$$
\pi_{0}(x)=\left(\pi_{-1}(x)+\pi_{1}(x)\right) / 2 .
$$

Let

$$
\Omega=\pi_{-1}([a, b]) \cup \pi_{0}([a, b]) \cup \pi_{1}([a, b]) .
$$

Then

$$
\Omega=\left[a, b+2 \sum_{x \in F} q(x)\right]-\bigcup_{x \in F}\left(\pi_{-1}(x), \pi_{0}(x)\right)-\bigcup_{x \in F}\left(\pi_{0}(x), \pi_{1}(x)\right) ;
$$

thus $\Omega$ is compact. The mappings $\pi_{-1}, \pi_{0}$, and $\pi_{1}$ have a common left inverse $\pi^{-1}: \Omega \rightarrow[a, b]$, so that $\pi^{-1}\left(\pi_{i}(x)\right)=x$ for $x \in[a, b]$ and $i \in\{-1,0,1\}$. Note $\pi^{-1}$ is increasing and nonexpansive.

We define functions $\hat{p}_{i}: \Omega \rightarrow[0,1], i \in \sigma^{k}, k=1,2, \ldots$ by

$$
\begin{aligned}
& \hat{p}_{i}\left(\pi_{0}(x)\right)=p_{i}(x), \\
& \hat{p}_{l}\left(\pi_{-1}(x)\right)=\lim _{y \rightarrow x-} p_{i}(y),
\end{aligned}
$$

and

$$
\hat{p}_{i}\left(\pi_{1}(x)\right)=\lim _{y \rightarrow x^{+}} p_{i}(y) .
$$

Lemma 1.1 and the summability condition (1.D) together imply that these one-sided limits exist. Note that the functions $\hat{p}_{1}$ are continuous on $\Omega$ and

$$
\sum_{i \in a^{k}} \hat{p}_{i} \equiv 1
$$

We define mappings $\hat{\beta}_{i}: \Omega \rightarrow \Omega, i \in \sigma^{k}, k=1,2, \ldots$ by

$$
\hat{\beta}_{i}\left(\pi_{0}(x)\right)=\pi_{0}\left(\beta_{i}(x)\right),
$$

and extending $\beta_{i}^{k}$ continuously to $\pi_{-1}([a, b])$ and $\pi_{1}([a, b])$. We then have $\pi^{-1} \circ \hat{\beta}_{i}=\hat{\beta}_{i} \circ \pi^{-1}$. For any continuous function $\phi$ on $[a, b]$ we can define a continuous function $\hat{\phi}$ on $\Omega$ by

$$
\hat{\phi}(\xi)=\phi\left(\pi^{-1}(\xi)\right) \text {. }
$$

Define the operator $\hat{T}: C(\Omega) \rightarrow C(\Omega)$ by

$$
\hat{T} \hat{\phi}(\xi)=\sum_{i \in \pi} \hat{p}_{i}(\xi) \hat{\phi}\left(\hat{\beta}_{i}(\xi)\right) ;
$$

we then have

$$
\hat{T}^{k} \hat{\phi}(\xi)=\sum_{i \in \sigma^{k}} \hat{p}_{i}(\xi) \hat{\phi}\left(\hat{\beta}_{i}(\xi)\right) .
$$

We now show that the family $\left\{\hat{T}^{k} \hat{\phi}\right\}$ is uniformly equicontinuous on $\Omega$. By Lemma 1.2 , we can choose a modulus of continuity $\lambda$ for $\left\{\phi \circ \beta_{i}: i \in \sigma^{k}, k=1,2, \ldots\right\}$ :

$$
\left|\phi\left(\beta_{i}(x)\right)-\phi\left(\beta_{i}(y)\right)\right|<\lambda(|x-y|)
$$

for all $i \in \sigma^{k}, k=1,2, \ldots$, and $x, y \in[a, b]$. Let $\xi, \eta \in \Omega$. Then

$$
\begin{aligned}
\left|\hat{T}^{k} \hat{\phi}(\xi)-\hat{T}^{k} \hat{\phi}(\eta)\right|= & \left|\sum_{i \in \sigma^{k}} \hat{p}_{i}(\xi) \hat{\phi}\left(\hat{\beta}_{i}(\xi)\right)-\hat{p}_{i}(\eta) \hat{\phi}\left(\hat{\beta}_{i}(\eta)\right)\right| \\
\leq & \left|\sum_{i \in \sigma^{k}} \hat{p}_{i}(\xi)\left[\hat{\phi}\left(\beta_{i}(\xi)\right)-\hat{\phi}\left(\hat{\beta}_{i}(\eta)\right)\right]\right| \\
& +\left|\sum_{i \in \sigma^{k}}\left[\hat{p}_{i}(\xi)-\hat{p}_{i}(\eta)\right] \hat{\phi}\left(\hat{\beta}_{i}(\eta)\right)\right| \\
\leq & \sum_{i \in \sigma^{k}} \hat{p}_{i}(\xi)\left[\hat{\phi}\left(\hat{\beta}_{i}\left(\pi^{-1}(\xi)\right)\right)-\hat{\phi}\left(\beta_{i}\left(\pi^{-1}(\eta)\right)\right)\right] \\
& +\|\hat{\phi}\| \sum_{i \in \sigma^{k}}\left|\hat{p}_{i}(\xi)-\hat{p}_{i}(\eta)\right| .
\end{aligned}
$$

where $\|\cdot\|$ denotes the supremum norm on $\Omega$ or $[a, b]$. Remember that the functions $\hat{p}_{i}$ are piecewise constant. Thus we have

$$
\sum_{i \in \sigma^{k}}\left|\hat{p}_{i}(\xi)-\hat{p}_{i}(\eta)\right| \leq \sum\left\{\operatorname{disc} p_{i}(x): x \in F \cap\left[\pi^{-1}(\xi), \pi^{-1}(\eta)\right]\right\} .
$$

From Lemma 1.1 it then follows that

$$
\sum_{i \in \pi^{k}}\left|\hat{p}_{1}(\xi)-\hat{p}_{i}(\eta)\right| \leq \sum\left\{M q(x): x \in F \cap\left[\pi^{-1}(\xi) \cdot \pi^{-1}(\eta)\right]\right\} .
$$

Our definition of $\Omega$ then implies

$$
\sum_{i \in \pi^{k}}\left|\hat{p}_{i}(\xi)-\hat{p}_{i}(\eta)\right| \leq M|\xi-\eta|
$$


Thus

$$
\left|\hat{T}^{k} \hat{\phi}(\xi)-\hat{T}^{k} \hat{\phi}(\eta)\right| \leq \lambda\left(\pi^{-1}(\xi)-\pi^{-1}(\eta)\right)+M\|\hat{\phi}\| \cdot|\xi-\eta| .
$$

This shows that the family $\left\{\hat{T}^{k} \hat{\phi}\right\}$ is uniformly equicontinuous on $\Omega$. By the Arzelà-Ascoli Theorem, it therefore has a uniformly convergent subsequence. Denote the limit of this sequence by $\hat{\phi}^{*}$. It is easy to show that $\|\hat{T} \hat{\psi}\| \leq\|\hat{w}\|$ for any function $\hat{y}$ on $\Omega$; thus

$$
\left\|\hat{T}^{k+1} \hat{\phi}-\hat{\phi}^{*}\right\|=\left\|\hat{T}^{k+1}\left(\hat{\phi}-\hat{\phi}^{*}\right)\right\| \leq\left\|\hat{T}^{k}\left(\hat{\phi}-\hat{\phi}^{*}\right)\right\|=\left\|\hat{T}^{k} \hat{\phi}-\hat{\phi}^{*}\right\| ;
$$

and $T^{k} \hat{\phi}$ converges uniformly to $\hat{\phi}^{*}$.

Since $T^{k} \phi=\left(\hat{T}^{k} \hat{\phi}\right) \circ \pi_{0},\left\{T^{k} \hat{\phi}\right\}$ converges uniformly to $\hat{\phi}^{*} \circ \pi_{0}$

1.4 Lemma. Let $X$ be a nonempty closed set in $[a, b]$ with $f^{-1}(X) \cup Z=X$, where $Z$ is finite. Then there exists a nonempty perfect subset $Y$ of $X$ with $f^{-1}(Y)=Y$.

Proof. Note that $X \cup Z$ contains the closure of the set of preimages of any given $x \in X$ under iterates of $f$; using this fact and hypothesis (iv) it can be shown that $X$ must be uncountable. Let $Y$ be the set of points whereof the intersection of every neighborhood with $X$ is uncountable. The complement of $Y$ is the union of all intervals open in $[a, b]$ with rational endpoints and whose intersection with $X$ is countable; this shows that $Y$ is closed and that $X-Y$ is countable. Hence $Y$ is nonempty. It is straightforward to show that $Y$ is a perfect set and $Y=f^{-1}(Y)$.

1.5 Lemma. Let $X$ be a nonempty closed set in $[a, b]$ with $f^{-1}(X) \cup Z=X$, where $Z$ is finite. Then there exists a continuous increasing map $h$ on $[a, b]$ with $h(X)=h([a, b])$ and such that

and

$$
\vec{f}=h \circ f \circ h^{-1}
$$

$$
\bar{p}_{i}=p_{i} \circ h^{-1}, i \in \sigma,
$$

satisfy hypotheses (i)-(iv) on $h([a, b])$.

Proof. By Lemma 1.4, $X$ contains a nonempty perfect set $Y$ with $f^{-1}(Y)=Y$. We construct $h:[a, b] \rightarrow[\inf Y, \sup Y]$ which maps $Y$ onto $[\inf Y$, sup $Y]$ as follows. Define $h(x)=\inf Y$ for $x \leq \inf Y$ and $h(x)=\sup Y$ for $x \geq \sup Y$. For each maximal open interval $(u, v)$ in $[a, b]-Y$, set $h(x)=(u+v) / 2$ for $x \in(u, v)$. We then extend $h$ continuously to the boundary of $Y$ and linearly to intervals contained in $Y$.

It is then straightforward to show that $\bar{f}$ and $\bar{p}_{i}$ satisfy hypotheses (i) (iv). One uses the fact that $Y=f^{-1}(Y)$, the continuity of $h$, and the fact that $h$ maps each interval not intersecting $Y$ to a single point.

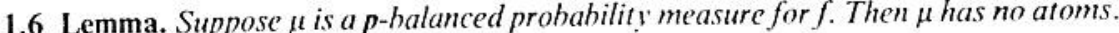

Proof. Recall that for any $i \in \sigma^{K}$ and any $x \in[a, b] . p_{i}(x) \leq P<1$. It follows that for any $i \in \sigma^{n K}$ and any $x \in[a, b], p_{i}(x) \leq P^{n}$. Since $\mu$ is invariant under $T^{n K}$, we have $\mu\{x\} \leq P^{n} \mu\left\{f^{n k}(x)\right\} \leq P^{n}$. Letting $n \rightarrow \infty$, we obtain the desired result.

1.7 Theorem. There exists a p-balanced probability measure $\mu$ for $f$.

Proof. The only difference between this theorem and Theorem 1.3 is the absence of the density hypothesis on $D$ as defined in Lemma 1.2. We now show how this hypothesis can be eliminated. Let $X$ be the closure of $D$. By Lemma 1.5, there exist hypothesis can be eliminated. Let $X$ be the closure of $D$. By continuous increasing a nonempty perfect set $Y$ cont $X$ with $f^{-1}(Y)=Y$ and a continuous increasing
function $h: Y \rightarrow[$ inf $Y$, sup $Y]$ so that $\bar{f}=h=f=h^{-1}$ and $\bar{p}_{i}=p=h^{-1}$ satisfy hypotheses (i)-(iv).

Set $\bar{C}=h(C)$; then $\bar{C}$ is the set of critical points of $\bar{f}$. Let

$$
\bar{D}=\bigcup_{n=0}^{\infty} f^{-n}(\bar{C}) \text {. }
$$

We now show that $\bar{D}$ is dense in $[\inf Y$, sup $Y]=h([a, b])$. Let $H \subset[\inf Y$. sup $Y]$ be a nonempty open interval. Since $h$ maps intervals not intersecting $Y$ to points, $h^{-1}(H) \cap Y$ and hence $h^{-1}(H) \cap X$ must be nonempty. Since $h^{-1}(H)$ is open, there exists $x \in h^{-1}(H) \cap D$. Then $f^{n}(x) \in C$ for some $n ; h\left(f^{n}(x)\right)=\bar{f}^{n}(h(x)) \in \bar{C}$ : and $h(x) \in H \cap \bar{D}$.

By Theorem 1.3, $\bar{f}$ has a $\bar{p}$-balanced probability measure $v$. By Lemma 1.6. $v$ has no atoms. We can thus define a probability measure $\mu$ on $[a, b]$ by

$$
\mu E=v h(E),
$$

and $\mu$ is a $p$-balanced measure for $f$. $\quad \square$

Finally we state a version of Theorem 1.7 with somewhat different hypotheses. In this version, the probabilities are not required to be piecewise-constant. but rather piecewise-Hölder-continuous. We require, however, that the map fbe expanding. We say $f$ is expanding if there exist constants $\alpha>0$ and $\lambda>1$ such that

$$
\left|\left(f^{n}\right)^{\prime}(x)\right|>x \lambda^{n}
$$

for all $n$ and all $x$ in the nonwandering set of $f$.

1.8 Theorem. Suppose $f$ is expanding on its nonwandering set and there exists a Hölder-continuous function $w$ on $[a, b]$ such that $p_{i}=w \circ \beta_{i}, i=1, \ldots, d$. Then there exists a p-halanced probability measure for $f$.

Proof. The operator $T$ in this case can be identified the Ruelle-Perron-Frobenius Proof. The operator $T$ in this case callow from a Ruelles P-F Theorem (Bowen 
[3, Theorem 1.7]): there exist $\lambda>0$, a continuous function $h$, and a probability measure $v$ on $[a, b]$ such that $L_{\log w} h=\lambda h, L_{\log w}{ }^{*} v=\lambda v, \int h d v=1$, and the sequence of functions $\lambda^{-n} L_{\log w}^{n} \phi$ converges uniformly to $h \int \phi d v$ for any continuous function $\phi$ on $[a, b]$. The condition $\sum p_{i} \equiv 1$ further implies $\lambda=1$ and $h \equiv 1$. Thus $v$ is the required $p$-balanced measure.

\section{Free energy and the maximal-entropy measure}

In this section we show an invariant measure has maximal entropy if and only if the free energy associated with its Jacobian is linear. Our result generalizes in some sense one of K. Zieman in [28]. Our techniques, however, differ from those used in [28]; we will use mainly ideas from Large-Deviation Theory to prove our main theorem.

We also show in the expanding case that a measure has maximal entropy if and only if its Jacobian has zero asymptotic variance. This fact was already known, but our proof follows from the results previously obtained in the beginning of paragraph two.

We first review the definition of a maximal-entropy measure. We denote by $M(f)$ the set of all probability measures invariant under $f$. We denote by $h(v, f)$ the entropy of $v \in M(f)$ under $f$. See Mañe [15] for definitions and general references on entropy. We further define the entropy of $f$, denoted $h(f)$, by

$$
h(f)=\sup _{v \in M(f)} h(v, f) .
$$

A most useful formula gives $h(f)$ as the asymptotic growth rate of the number of preimages of a point:

$$
h(f)=\lim _{n \rightarrow \infty} \frac{1}{2} \log \# f^{-n}(x) .
$$

If $\mu \in M(f)$ and $h(\mu f)=h(f)$, we call $\mu$ a maximal-entropy measure for $f$. Newhouse and Yomdim [26] showed that a maximal-entropy measure exists for any $C^{\infty}$ map. Hofbauer [8] showed that in the case $d=2$ (unimodal map) the maximal-entropy measure is unique. Polynomial maps of higher degree may have more than one maximal-entropy measure.

Let $v$ be a Borel measure on $[a, b]$. We define the Jacobian of $v$ to be a function $J_{v}:[a, b] \rightarrow[0, \infty]$ given by

$$
J_{v}(x)=\lim _{\varepsilon \rightarrow 0} \frac{v f([x-\varepsilon, x+\varepsilon])}{v[x-\varepsilon, x+\varepsilon]} .
$$

Alternatively, $J_{v}$ is the Radon-Nikodym derivative of $v \circ f$ with respect to $v$; this shows that $J_{v}(x)$ is defined for $v$-almost all $x$ in $[a, b]$.

If $v \in M(f)$ then $J_{v}$ must be positive $v$-almost everywhere; moreover.

$$
\sum_{f(y)=x} \frac{1}{J_{v}(y)}=1
$$

for $v$-almost all $x$ in $[a, b]$. Moreover, if $v$ is a weight-balanced measure associated with probabilities $\left\{p_{i}: i \in \sigma\right\}$, and $\left\{\beta_{i}: i \in \sigma\right\}$ are the corresponding inverse branches of $f$, then we have

$$
\text { (2.A) } \quad p_{i}(x)=\frac{1}{J_{v}\left(\beta_{i}(x)\right)}
$$

for $v$-almost all $x$ in the domain of $\beta_{i}$. If $J_{v}(x)>0$ for $y$-almost all $x$ then one can show $h(v)=\int \log J_{v}(x) d v(x)$ (as shown in [11]).

We consider $v \in M(f)$ with Jacobian $J_{v}$. Let $\beta_{1}, \ldots, \beta_{d}$ denote the inverse branches of $f$. We define functions $p_{1}, \ldots, p_{d}$ by (2.A). For $i \in \sigma^{k}$, we define $\beta_{i}$ and $p_{i}$ recursively as in the previous section. We define functions $w_{n}^{t}, n=1,2, \ldots, t \in \mathbb{R}$ by $k_{n}^{t}(x)=$ $p_{i}\left(f^{n}(x)\right)^{t}$, where $i \in \sigma^{n}$ is chosen so that $x=\beta_{i}\left(f^{n}(x)\right)$. It easily verified that the functions $w_{n}$ satisfy the following relations:

$$
w_{n}^{t}(x)=w_{n-k}^{t}\left(f^{k}(x)\right) w_{k}^{t}(x), 1 \leq k<n
$$

$$
\begin{aligned}
& w_{1}^{t}(x)=\left(J_{v}(x)\right)^{-t} ; \\
& w_{n}^{t}(x)=\prod_{k=1}^{n-1} w_{1}^{t}\left(f^{k}(x)\right)=\prod_{k=1}^{n-1} J_{v}\left(f^{k}(x)\right)^{-t} .
\end{aligned}
$$

2.1 Definition. For $v \in M(f)$, we define the free encrgy of $J_{v}$ to be a function $c_{v}: \mathbb{R} \rightarrow[0, \infty]$ given by

$$
c_{v}(t)=\lim _{k \rightarrow \infty} \frac{1}{k} \log \int w_{k}^{-t}(x) d v(x)
$$

when this exists.

We say that a measure $v$ is in $M(f)^{*}$ if the above limit exists and the three conditions below are satisfied:

1) $v$ is balanced, that is for any $\phi$ continous function and any $\mu$ probability measure. we have

$$
\int \phi d v=\lim _{n \rightarrow \infty} T^{n} \phi d \mu .
$$

2) $c_{v}(t)$ is differentiable by parts and right and left derivatives exist for the values of $t$ where $c_{v}(t)$ is not differentiable. We also assume $c_{v}$ is differentiable at $t=0$.

3) For each value of $t$ the Ruelle-Perron-Frobenius Operator associated to $\log w_{1}^{1-t}(x)$ is uniformily equicontinuous.

We point out that all the above properties 1), 2), 3) are true, and the above limit exists in the case where the Jacobian is Hölder-continuous and $f$ is expanding. The function $c_{v}(t)$, in this case is in fact real analytic.

Examples of situations where the free-energy (also called pressure) is not differentiable but differentiable by parts appear in ([11] (page 95). [13] (page 402) 
[14] (Theorem 1)). Examples where the pressure is differentiable but the potential is not Hölder-continuous appear in Theorem 1 in [14], under the condition $1<\gamma<2$ (see [14]).

Remark. In the paper of F. Hofbauer-"Examples for the Nonuniqueness of the Equilibrium State"-Transactions AMS vol. 228, 1977, pp. 223-241) the shift in two Equilibrium State"-Transactions A potential is analyzed and the author shows that for the Ruelle-Perron-Frobenius Operator property 3 ) above is true (see second paragraph from mentioned paper and in some of them, two equilibrium states can coexist (see table in page 239). If one considers for example, the class of examples satisfying a), b) and c) on page 238 in the paper of Hofbauer, then one can find a large class of situations on page 238 in the pat is a nontrivial where two equilibrium states coexist, one is a Dirac-Delta and the other is a nobian of the above measure $\mu$. In this case the potential given by this Jacobian produces the same above measure $\mu$. In the can $p(t)$ is presented in fig. 1 in page 402 in property 3 ) above. The graph of the function 2 ) above. Therefore the hypothesis of the last mentioned paper and it satisfies 2) above. Therefore the hypothesis of Theorem 2.2 below cover cases of weight-balanced measures not represented only by the expanding case with Hölder-continuous potential (or Jacobian) and we can ask if a balanced measure of this kind can be the maximal measure. The answer is no, because $p(t)$ is not linear.

The purpose of presenting all the above examples is to stress the fact that in several situations the free-energy (or pressure) is not so nice as in the expanding maps and Hölder-continuous case. Nevertheless under some suitable assumptions (to be in $\left.M(f)^{*}\right)$ we will be able to show in Theorem 2.2 some properties for $c_{v}(t)$.

In [11], [19], [23] several examples of the pathologies of the non-expanding case are considered.

We do not use in our proof properties like Quasi-Compactness of Operators ([1], [19], [23]), or the approach of [22].

The concept of free energy comes from Large-Deviation Theory. The main concepts of this theory are typically defined not for all objects of interest, but for a dense subset thereof. In this case we require $v \in M(f)^{*}$ rather than $v \in M(f)$. Ellis a dense subset thereof. In this case we require $v \in M(f)$ rather than Legendre-Fenchel transform.

Our main goal in this section is the following:

2.2 Theorem. Let $v \in M(f)^{*}$, that is:

1) $v$ is halanced; that is, for any continous function $\phi$ and any prohahility measure $\mu$, we have

$$
\int \phi d v=\lim _{n \rightarrow x} T^{n} \phi d \mu
$$

2) $c_{v}(t)$ is differentiable by parts and there exist right and left deritatives at the ralues of $t$ where $c_{v}(t)$ is not differentiable. We also assume $c_{\mathrm{v}}$ is differentiable at $t=0$.

3) For each value of the Ruelle-Perron-Frobenius Operator associated with $\log w_{1}^{1-t}(x)$ is uniformly equicontinuous.

Then $h(v, f)=h(f)$ if and only if $c_{v}$ is a linear function.

Before the proof of the theorem we need a lemma that relates pressure and free energy (see [22] for the concept of pressure and about Ruelle-Perron-Frobenius operators).

2.3 Lemma. For fixed $x \in[a, b]$, we have

$$
c_{v}(t)=\lim _{k \rightarrow \infty} \frac{1}{k} \log \sum_{i \in \pi^{k}} p_{i}(x)^{1-t} .
$$

This limit converges and hence $c_{v}(t)$ is defined for all real $t$.

Note: It should be understood here that the sum includes only those terms with $p_{i}(x) \neq 0$, as we consider cases with $t \geq 1$.

Proof. Let the operator $T$ be defined as in (1.A). As the measure is balanced

$$
\int \phi d v=\lim _{n \rightarrow \infty} \int T^{n} \phi d \mu
$$

for any fixed probability measure $\mu$ (not necessarily invariant) and any continuous function $\phi$. See also the definition of an attractive measure in [2]. We consider the particular case where $\phi(x)=w_{m}^{-1}(x)$ and $\mu$ is a unit point mass at $x$. We then have

$$
\int w_{m}^{-t}(x) d \mu(x)=\lim _{n \rightarrow x} T^{n} w_{m}^{-t}(x)
$$

Thus

(2.C) $\quad c_{v}(t)=\lim _{m \rightarrow \infty} \frac{1}{m} \log \lim _{n \rightarrow \infty} T^{n} w_{m}^{-t}(x)$.

We assume $n>m$ without loss of generality. Recalling (1.A), we have

$$
T^{n} w_{m}^{-t}(x)=T^{n-m} T^{m} w_{m}^{-t}(x)=\sum_{i \in \sigma^{n-m}} p_{i}(x)\left(T^{m} w_{m}^{-t}\right)\left(\beta_{i}(x)\right) .
$$

From (2.B), we have

$$
\begin{aligned}
\left(T^{m} w_{m}^{-t}\right)\left(\beta_{i}(x)\right) & =\sum_{j \in \tau m^{\prime}} w_{m}^{-t}\left(\beta_{j}\left(\beta_{i}(x)\right)\right) p_{j}\left(\beta_{i}(x)\right) \\
& =\sum_{j \in \pi^{m}} w_{m}^{1-t}\left(\beta_{j}\left(\beta_{i}(x)\right)\right)=\sum_{j \in \sigma m} p_{j}\left(\beta_{i}(x)\right)^{1-t} .
\end{aligned}
$$


since from the definition of $w_{m}^{-t}$ we have $p_{j}\left(\beta_{i}(x)\right)=w_{m}^{1}\left(\beta_{j}\left(\beta_{i}(x)\right)\right)$. Using (2.B), and setting $\psi(x)=\log w_{1}^{1-t}(x)$, we find

$$
\left(T^{m} w_{m}^{-t}\right)(y)=\sum_{j \in \sigma m} w_{m}^{1-t}(y)=L_{w}^{m}(1)(y),
$$

where $L_{v}$ is the Ruelle-Perron-Frobenius operator associated with $w$. Because of the uniform equicontinuity of iterates of $L_{w}^{m}$, we can find a constant $K>0$ such that

(2.D) $\quad e^{-K} L_{\psi}^{m}(1)(x)<L_{\psi}^{m}(1)\left(\beta_{i}(x)\right)<e^{K} L_{\psi}^{m}(1)(x)$.

We can interpret this as

$$
e^{-\kappa} T^{m} w_{m}^{-t}(x)<T^{m} w_{m}^{-t}\left(\beta_{i}(x)\right)<e^{K} T^{m} w_{m}^{-t}(x) .
$$

Since $\sum p_{i}(x) \equiv 1$, we have finally

$$
e^{-\kappa} T^{m} w_{m}^{-t}(x)<T^{n-m} T^{m} w_{m}^{-t}(x)<e^{K} T^{m} w_{m}^{-1}(x) .
$$

Equation (2.C) now easily transforms to

$$
\begin{aligned}
c_{v}(t) & =\lim _{m \rightarrow \infty} \frac{1}{m} \log T^{m} w_{m}^{-t}(x) \\
& =\lim _{m \rightarrow \infty} \frac{1}{m} \log \sum_{i \in \sigma^{m}} w_{m}^{-t}\left(\beta_{i}(x)\right) p_{i}(x) \\
& =\lim _{m \rightarrow \infty} \frac{1}{m} \log \sum_{i \in \sigma m} p_{i}(x)^{-t} p_{i}(x),
\end{aligned}
$$

using once more the definition of $w_{m}^{-t}$.

To show now convergence of the limit, we write $i \in \sigma^{m}$ as a concatenation $i=j \mid k$, for some $j \in \sigma^{n}$ and $k \in \sigma^{m-n}$. Thus

$$
\sum_{i \in \sigma^{m}} p_{i}(x)^{1-t}=\sum_{j \in \sigma^{n}} \sum_{\boldsymbol{k} \in \sigma^{m-n}} p_{\boldsymbol{k}}(x)^{1-t} p_{j}\left(\beta_{k}(x)\right)^{1-t} .
$$

using (1.B). Note now that (2.D) can also be interpreted as

$$
e^{-K} \sum_{j \in \sigma^{n}} p_{j}(x)^{1-t}<\sum_{j \in \sigma^{n}} p_{j}\left(\beta_{k}(x)\right)^{1-t}<e^{K} \sum_{j \in \sigma^{n}} p_{j}(x)^{1-t} .
$$

Thus

$$
e^{-K}<\frac{\sum_{j \in \sigma^{n}} p_{j}(x)^{1-t} \sum_{k \in \sigma^{m-n}} p_{k}(x)^{1-t}}{\sum_{i \in \sigma^{m}} p_{i}(x)^{1-1}}<e^{\kappa}
$$

$$
\left|\log \sum_{j \in \sigma^{n}} p_{j}(x)^{1-t}+\log \sum_{k \in \sigma^{m}-n} p_{k}(x)^{1-t}-\log \sum_{i \in \sigma^{m}} p_{i}(x)^{1-t}\right|<K .
$$

It then follows by a standard subadditive argument (used for example in Lopes [10]) that the limit converges.

Proof of Theorem 2.2. First we assume $c_{v}$ is not linear and show $h(v)$ has less than maximal entropy. From Lemma 2.3, we have

$$
c_{v}(1)=\lim _{n \rightarrow \infty} \frac{1}{n} \log \sum\left\{1: i \in \sigma^{n} ; p_{i}(x)>0\right\},
$$

$x \in[a, b]$ being arbitrary. Note $p_{i}(x)>0$ if and only if $\beta_{i}(x)$ is defined. Let us assume $x \notin F$, where $F$ is as defined in Section 1 ; so that $\beta_{i}(r)=\beta_{j}(x)$ only if $i=j$. Then we have $\sum\left\{1: i \in \sigma^{n} ; p_{i}(x)>0\right\}=\# f^{-n}(x)$, the number of preimages of $x$ under $f^{n}$. Thus

$$
c_{v}(1)=\lim _{n \rightarrow \infty} \frac{1}{n} \log \# f^{-n}(x)=h(f) .
$$

Now define $C_{v}$ to be the Legendre-Fenchel transform of $c_{v}$ :

$$
C_{\mathrm{v}}(z)=\sup _{t \in \mathbb{Q}}\left(t z-c_{\mathrm{v}}(t)\right) .
$$

Recall $c_{v}(0)=0$; thus $C_{v}$ is nonnegative.

From Definition 2.1 we find

$$
\begin{aligned}
c_{v}(t) & =\lim _{k \rightarrow \infty} \frac{1}{k} \log \int w_{k}^{-t} d v(x) \\
& \geq \lim _{k \rightarrow \infty} \frac{1}{k} \int \log w_{k}^{-t} d v(x) \\
& =\lim _{k \rightarrow \infty} \frac{1}{k} \int \sum_{j=1}^{k-1} t \log J_{v}\left(f^{j}(x)\right) d v(x) \\
& =\int t \log J_{v}(x) d v(x)=\operatorname{th}(v) .
\end{aligned}
$$

using (2.B) in the next-to-last step and the invariance of $v$ in the last. Thus $c_{v}(t)-t h(v) \geq 0$ and $C_{v}(h(v))=0$ (recall $C_{v}$ is nonnegative).

Let us assume now that $C_{v}$ is not linear. In this case it can be shown that $C_{v}$ is finite and differentiable in a neighbourhood of 0 (see page 218 in [4]). Since $C_{v}$ attains its minimum at $h(v)$, we have $C_{v}^{\prime}(h(v))=0$.

The Legendre-Fenchel transform can be easily inverted:

$$
c_{v}(t)=\sup _{z \in}\left(z-t C_{v}(z)\right) .
$$

In particular,

$$
\sup _{z \in \delta}\left(z-C_{v}(z)\right)=c_{v}(1)=h(f) \text {. }
$$


But

$$
\frac{d}{d z}\left(z-C_{v}(z)\right)=1
$$

for $z=h(v)$, so this is not a local maximum for $z-C_{\mathrm{v}}(z)$. Thus

$$
h(v)=h(v)-C_{v}(h(v))<h(f) .
$$

Now we assume $c_{v}$ is linear and show that $h(v) \geq h(f)$. We fix a point $x \in[a, b]-F$ and define a sequence of probabilities $v_{n}, n=0,1, \ldots$ by

$$
\int \phi d v_{n}(x)=T^{n} \phi(x)
$$

for any continuous function $\phi$ on $[a, b]$. Note that $v_{n}$ is supported on $f^{-n}(x)$ and for any subset $S$ of $f^{-n}(x)$ we have

$$
v_{n} S=\sum_{y \in S} w_{n}^{1}(y) .
$$

Consider a certain value $x$ fixed. Let $\varepsilon>0$ be given. We define a sequence of closed sets $B_{n}: n=1,2,3, \ldots$ by

$$
B_{n}=\left\{y \in f^{-n}(x) \text { such that }\left|-\frac{1}{n} \log w_{n}(y)-h(v)\right| \geq \varepsilon\right\} .
$$

Define also $A_{n}$ by $A_{n}=f^{-n}(x)-B_{n}$

Our chief tool in this argument is [4, Theorem II.6.1], which says that

$$
\limsup _{n \rightarrow \infty} \frac{1}{n} \log v_{n} B_{n} \geq-\inf _{|:-h(v)| \geq \varepsilon} C_{v}(z) \text {. }
$$

We now establish

$$
\lim _{n \rightarrow \infty} \frac{1}{n} \log \# A_{n}=h(f)
$$

Since $c_{v}$ is linear, $c_{v}^{\prime} \equiv c_{v}(1)-c_{v}(0)=h(f)$; thus $C_{v}(h(f))=0$ while $C_{v}(z)=\infty$ for $z \neq h(f)$. Thus from (2.E) we have

$$
\lim _{n \rightarrow \infty} \frac{1}{n} \log v_{n} B_{n}=-\infty .
$$

By assumption, $J_{v}$ is bounded; from (2.B) we find that

$$
v_{n} B_{n}=\sum_{y \in B_{n}} w_{n}^{1}(y) \geq\left(\# B_{n}\right)\left(\sup J_{v}\right)^{-n} .
$$

Thus

$$
\lim _{n \rightarrow,} \frac{1}{n} \log \# B_{n}=-\infty
$$

Note that as the cardinal of $B_{n}$ is a natural number, the only possibility is that $B_{n}$ is eventually empty.

Hence

$$
\begin{aligned}
\lim _{n \rightarrow \infty} \frac{1}{n} \log \# A_{n} & =\lim _{n \rightarrow \infty} \frac{1}{n} \log \left(\# A_{n}+\# B_{n}\right) \\
& =\lim _{n \rightarrow \infty} \frac{1}{n} \log \# f^{-n}(x)=h(f) .
\end{aligned}
$$

Note that $w_{n}^{1}(y)>e^{-\{h(v)+\varepsilon) n}$ for $y \in A_{n}$. Thus

$$
\# A_{n} \leq e^{(h(v)+\varepsilon) n} v_{n} A_{n} \leq e^{(h(v)+\varepsilon) n} .
$$

Thus we have

$$
h(f)=\lim _{n \rightarrow \infty} \frac{1}{n} \log \# A_{n} \leq h(v)+\varepsilon .
$$

Since $\varepsilon>0$ is arbitrary, this proves the theorem.

Suppose now that $f$ is expanding. We will show a proof of a result already known [1], [9], [23], [21].

2.4 Lemma. $c_{v}(t)$ is a $C^{2}$ function of $t$

Proof. This follows from the same type of argument as those used by Mañe [15. Corollary 1.4] or Pollicot [20] for the pressure function. We conjugate $f$ to a Markov shift of finite type and consider a complexification of $t$ in the integrand $w_{k}^{-t}(x)$ in the definition of $c_{v}$. The hypotheses that $f$ is expanding and $J$ is Hölder-continuous are important here.

2.5 Definition. Let $v \in M(f)$ and $\phi:[a, b] \rightarrow \mathbb{R}$ be continuous we define the asymptotic variance of $\phi$, denoted $\sigma_{\phi}^{2}$, by

$$
\sigma_{\phi}^{2}=\lim _{n \rightarrow \infty} \frac{1}{n} \int\left(n \int \phi(y) d v(y)-\sum_{j=0}^{n-1} \phi\left(f^{j}(x)\right)\right)^{2} d v(x) .
$$

Przytycki, Urbanski and Zdunik [21, Lemma 1] showed that this limit exists and further that $\sigma_{\phi}^{2}=0$ if and only if $\phi$ is cohomologous to zero.

2.6 Theorem. Let $v \in M(f)^{*}$. Then

$$
\sigma_{\log J_{v}}^{2}=0
$$

if and only if $h(v)=h(f)$. 
Proof. By Lemma 2.4, $c_{v}$ can be differentiated under the limit; thus we obtain

$$
c_{v}^{\prime}(t)=\lim _{n \rightarrow \infty} \frac{\int-w_{n}^{-t}(x) \log w_{n}^{1}(x) d v(x)}{n \int w_{n}^{-t}(x) d v(x)}
$$

and

$$
c_{v}^{\prime \prime}(t)=\lim _{n \rightarrow \infty} \frac{\int w_{n}^{-t}(x) d v(x) \int w_{n}^{-t}(x)\left(\log w_{n}^{1}(x)\right)^{2} d v(x)-\left(\int w_{n}^{-t}(x)\left(\log w_{n}^{\prime}(x)\right) d v(x)\right)^{2}}{n\left(\int w_{n}^{-t}(x) d v(x)\right)^{2}}
$$

Thus, using (2.B) and the invariance of $v$, we find

$$
\begin{aligned}
c_{v}^{\prime \prime}(0) & =\lim _{n \rightarrow \infty} \frac{1}{n}\left(\int\left(\log w_{n}^{1}(x)\right)^{2} d v(x)-\left[\int \log w_{n}^{1}(x) d v(x)\right]^{2}\right) \\
& =\lim _{n \rightarrow \infty} \frac{1}{n}\left(\int\left[\sum_{j=0}^{n-1} \log J_{v}\left(f^{j}(x)\right)\right]^{2} d v(x)-\left[n \int \log J_{v}(x) d v(x)\right]^{2}\right) .
\end{aligned}
$$

From the theory of large deviations it is known that $c_{v}^{\prime \prime}(0)=0$ if and only if $c_{v}$ is linear. From Theorem 2.2, this occurs only if $h(v)=h(f)$.

\section{Entropy of a weight-balanced measure: an example}

For a map with essentially the same number of preimages for each point, the maximal-entropy measure can be easily described as a weight-balanced measure corresponding to equal weights on all branches (with multiplicity) of the inverse. When the number of preimages varies from point to point, there is no known explicit formula for the weights which yield a measure of maximal entropy. In this section we present an example of such a map whose entropy is explicitly calculable. This example shows that maximal entropy is not necessarily attained by assigning equal weight to each branch of the inverse map when the number of branches depends on the point.

We define $f:[0,1] \rightarrow[0,1]$ by

$$
f(x)= \begin{cases}2 x+\frac{1}{4} & \text { if } \quad 0 \leq x \leq \frac{3}{8}, \\ \frac{7}{4}-2 x & \text { if } \frac{3}{8} \leq x \leq \frac{3}{4} \\ 1-x & \text { if } \frac{3}{4} \leq x \leq 1 .\end{cases}
$$

Note that Lebesgue measure on $[0,1]$ is an invariant probability measure for this map.

We set $X=(0,1 / 4), Y=(1 / 4,3 / 4)$, and $Z=(3 / 4,1)$. Any point in $X$ has just one preimage in $Z$. Any point in $Y$ has one preimage in $Y$ and one preimage in $X$ Any point in $Z$ has two preimages in $Y$. Thus if $x \in X \cup Y \cup Z$ and we set $\xi_{n}=\#\left(f^{-n}(x) \cap X\right), \eta_{n}=\#\left(f^{-n}(x) \cap Y\right)$, and $\zeta_{n}=\#\left(f^{-n}(x) \cap Z\right)$, then we have the formula

$$
\left(\begin{array}{l}
\xi_{n+1} \\
\eta_{n+1} \\
\zeta_{n+1}
\end{array}\right)=\left(\begin{array}{lll}
0 & 1 & 0 \\
0 & 1 & 2 \\
1 & 0 & 0
\end{array}\right)\left(\begin{array}{l}
\xi_{n} \\
\eta_{n} \\
\zeta_{n}
\end{array}\right)
$$

The asymptotic growth rate of the number of preimages of a point is thus given by the largest eigenvalue of the matrix in this equation: this is

$$
\lambda=\frac{(224+24 \sqrt{87})^{1 / 3}+(224-24 \sqrt{87})^{1 / 3}+2}{6} \doteq 1.6956
$$

The entropy of $f$ is then given by $\log \lambda \doteq 0.52805$

Lebesgue measure $m$ is an invariant measure for $f$ : its Jacobian under $f$ is equal to 2 on $(0,3 / 4)$ and equal to 1 on $(3 / 4,1)$. The corresponding weights are $1 / 2$ on each branch when there are two inverse branches, and 1 (necessarily) when there is one inverse branch.

We can calculate the entropy of $m$ under $f$ explicitly as $h(m)=\int \log J_{m}(x) d m(x)$ We find $h(m)=\log 2 \cdot 3 / 4 \doteq 0.51986<h(f)$.

\section{References}

[1] Baladi, V. and Keller, G.: Zeta Functions and transfer operators for piecewise monotone transformations. Comm. Math. Phys. 127 (1990), 459-478

[2] Barnsley. M. F.. Demko. S. G., Elton, J.H., Geronimo, J.S.: Markov processes arising from function iteration with place-dependent probabilities. Anal. Inst. Henri Poincar 24, 3 (1988), 367-394

[3] Bowen, R.: Equilibrium states and the ergodic theory of Anosov diffeomorphisms. Springer-Verlag

[4] Ellis, R.S.: Entropy, large deviations, and statistical mechanics. Springer-Verlag 1985

[5] Elton. J.H.: An ergodic theorem for iterated maps. Ergodic Theory Dynamical Systems 7 (1987), $481-488$

[6] Elton, J.H.: A multiplicative ergodic theorem for Lipschitz maps (preprint)

[7] Freire, A., Lopes, A. O. and Mañe, R.: An invariant measure for rational maps. Bol. Soc. Brasil Mat. 14 (1983), 45-62

[8] Hofbauer, F.: On intrinsic ergodicity of piecewise-monotone transformations with positive entropy IL. Israel J. Math. 38, 1-2 (1981), 107-115

[9] Hofbaver. F and Keller. G. Ergodic Properties of invariant measures for piecewise monotone transformations. Math. Z. 180 (1982) 119-140

10] Lopes. A. O.: Entropy and large deviation. Nonlinearity 3 (1990), 527-546

[11] Lopes. A. O.: A dimension spectra and mathematical model for phase transition. Adv, in Appl. Math. 11. 4 (1990), 475-502

[12] Lopes, A.O and Withers, W.D. Differentiability of the Hausdorff dimension of weight-balanced measures (preprint)

[13] Lopes. A.: A First Order Level-2 Phase Transition in Thermodynamic Formalism. J. Statist. Phys. 60. 3/4 (1990), 395-411

[14] Lopes, A.: The Zeta Function, nondifferentiability of pressure and the critical exponent of transition (to appear in Adv. in Math) 
5] Mañe, R.: The Hausdorff dimension of horseshoes of diffeomorphisms of surfaces. Bol. Soc. Brasil. Mat (to appear)

[16] Misiurewicz, M.: Absolutely continuous measures for certain maps of the interval. Publ. Math. IHES 53 (1981), 17-51

[17] Misiurewicz, M. and Szlenk, W.: Entropy of piecewise-monotone maps. Astérisque 50 (1977), 299-310

[18] Misiurewicz, M. and Szlenk, W.: Entropy of piecewise-monotone maps. Studia Math. 67 (1980), 45-63

[19] Nowiki, T. and van Strien, S.: Invariant measures exist under a summability condition for unimodal maps (to appear in Invent. Math.)

[20] Pollicot, M.: A complex Ruelle-Perron-Frobenius operator theorem and two counterexamples. Ergodic Theory Dynamical Systems 4 (1984), 135-146

[21] Przyztycki. F., Urbanski, M. and Zdunik, A.: Harmonic, Gibbs, and Hausdorff measure on repellers for holomorphic maps. Annals of Math. 130 (1989), 1-40

[22] Ruelle, D.: Thermodynamic formalism. Addison-Wesley 1978

[23] Rychlik, M.: Bounded variation and invariant measures. Studia Math. LXXVI (1983). 69-80

[24] Servizi, G., Turchetti, G. and Vaienti, S.: Generalized dynamic variables and measures for the Julia Set. Nouvo Cimento (to appear)

[25] Withers, W.D.: Differentiability with respect to parameters of average values in probablistic contracting dynamical systems. Ergodic Theory Dynamical Systems 10 (1990), 599-610

[26] Yomdim, Y.: Volume growth and entropy. Israel J. Math. 57, 3 (1987). 285-300

[27] Zieman, K.: Almost sure invariance for some maps of the interval. Ergodic Theory Dynamical Systems 5 (1985), 625-640

[28] Zieman, K.: Refinement of Shannon-McMillan-Breiman theorem for some maps of the interval (preprint)

Received April 2, 1991. Revised July 1, 1991. In final form December 20, 1991

Artur O. Lopes, Instituto de Matematica, Universidade Federal do Rio Grande do Sul. Av. Bento Goncalves, 9500. Porto Alegre-RS-91500-Brasil

Wm. Douglas Withers, Department of Mathematics, United States Naval Academy. Annapolis, MD 21402, USA

\section{Metric properties of positively ordered monoids}

Friedrich Wehrung

(Communicated by Rüdiger Göbel)

Abstract. We introduce here an intrinsic (quasi-) metric on each positively ordered monoid (P.O.M.), which is defined in terms of the evaluation map from the given P.O.M. to its bidual and for which P.O.M. homomorphisms are continuous. Moreover, we find a class of refinement P.O.M.'s which, equipped with the canonical metric, are complete metric spaces; this class includes the class of weak cardinal algebras, but also most cases of completions of a certain kind (we will call it 'strongly reduced products') of P.O.M.'s, and of which a prototype has been used in a previous paper for the description of the evaluation map of a given refinement P.O.M. This result can also be viewed as a wide generalization to the non-linearly ordered case (for example weak cardinal algebras) of the (Cauchy-) completeness of the real line.

1991 Mathematics Subject Classification: 06F05. 06F30; 28B10.

\section{Introduction; basic definitions and notations}

We recall here the definition of positively ordered monoids. as it appears in [15].

Definition. A positively ordered monoid (from now on a P.O.M.) is a structure $(A,+, 0, \leq)$ where $(A,+, 0)$ is a commutative monoid and $\leq$ is a preordering of $A$ such that $(A,+, 0, \leq)$ satisfies the following:

(i) $\quad(\forall a, b, c)(a \leq b \Rightarrow a+c \leq b+c)$

(ii) $\quad(\forall a)(a \geq 0)$.

In particular, when $(A,+, 0, \leq)$ is a commutative monoid, then we can define a preordering (not always antisymmetric) $\leq$ on $A$ by

$$
(\forall x, y \in A)(x \leq y \Leftrightarrow(\exists z \in A)(x+z=y)) .
$$

